\title{
Design and Characterization of an MFSK-Based Transmitter/Receiver for Ultrasonic Communication Through Metallic Structures
}

\author{
Thomas Hosman, Mark Yeary, Senior Member, IEEE, and John K. Antonio, Senior Member, IEEE
}

\begin{abstract}
Some applications require wireless transmission of information to and from devices located inside metal enclosures, e.g., a closed shipping container in transit. However, traditional radio frequency (RF) communication schemes are not capable of transmitting signals through metal enclosures. As an alternative to RF, an ultrasonic communication system based on multitone frequency-shift keying (MFSK) has been developed and evaluated using the steel corner posts of shipping containers as the communication medium. Empirical studies have been performed on the steel channel. The communication system is configurable and consists of two or more modules. The modules are mounted to the metal posts and utilize an inexpensive ultrasonic transducer to send and receive modulated signals through the metal channel. A module also makes use of an inexpensive digital-signal-processing chip for modulating and demodulating MFSK signals. Two case studies for the shipping container application were evaluated: 1) communicating through one container and 2) communicating between stacked containers. Regarding the second case study, a key discovery was that each of the aggregate steel columns formed by a stack of shipping containers can serve as an ultrasonic communication channel that spans all containers in the stack.
\end{abstract}

Index Terms-Channel characterization, multitone frequencyshift keying (MFSK), shipping containers, ultrasonic communication.

\section{INTRODUCTION}

$\mathbf{M}$ UCH international shipping is carried in standardized steel containers, which can be transferred by ship, truck, or rail. It is of great importance to both shippers and security professionals to gather information about the contents (e.g., shipping container's serial number, radio-frequency identification (RFID) tags, and information about the container's state, such as temperature and humidity sensor readings within such containers). Closed shipping containers act as Faraday cages, which preclude the use of traditional radio-frequency (RF) communication schemes for transmitting data in and out

Manuscript received October 18, 2010; revised January 21, 2011; accepted March 9, 2011. Date of publication June 7, 2011; date of current version November 9, 2011. This work was supported by the U.S. Federal Highway Administration through Grant SAFTEA-LU 1934 and Grant SAFTEA-LU 1702. The Associate Editor coordinating the review process for this paper was Dr. Jesús Ureña.

T. Hosman and M. Yeary are with the School of Electrical and Computer Engineering, University of Oklahoma, Norman, OK 73019 USA.

J. K. Antonio is with the School of Computer Science, University of Oklahoma, Norman, OK 73019 USA.

Color versions of one or more of the figures in this paper are available online at http://ieeexplore.ieee.org.

Digital Object Identifier 10.1109/TIM.2011.2149270 of these metal enclosures. Ultrasound, however, presents a promising alternative. Steel is an excellent conduit of ultrasonic energy, and ultrasound transmission requires no compromise of the container's security or structural integrity. Therefore, the focus of this paper is to present empirical results for a digital transmitter/receiver system for a new method of ultrasonic communication for metal shipping containers.

Shipping containers are typically either 20 or $40 \mathrm{ft}$ long, $8 \mathrm{ft}$ wide, and $81 / 2 \mathrm{ft}$ tall. They are constructed with four structural steel corner posts, which terminate at the top and bottom with cast steel blocks. As many as 12 containers can be stacked in shipyards or on board ships. When stacked, the weight of the containers and their cargo is carried by each of the continuous columns of the four corner posts. For structural and security reasons, the doors of a container must not be opened in transit, and holes may not be drilled through a container's walls.

Because traditional wireless RF communication systems cannot be applied for communicating through closed shipping containers, ultrasonic communication methods were explored by the authors of this paper. The basic architecture includes mounted modules to establish two-way communication. Future modules will also be capable of participating in a relay network along an aggregate column formed by a stack of containers. In one use-case scenario, one module would be fixed and mounted inside a container, and the other would be handheld, portable, and held in contact with a container by an operator. Modules mounted inside a container may use RF to communicate with RFID tags and/or sensor modules that are also inside the container.

Ultrasonic sensor systems have been known within the instrumentation community for many years. Common applications of ultrasonic systems include range finding, flow rate determination, and monitoring of chemical processes [1], [2]. The most common applications of ultrasonics do not involve ultrasonic communications; rather, they originate from the relatively matured fields of nondestructive testing and medical imaging [3], [4]. For example, in the field of nondestructive testing, the propagation of high-frequency (several megahertz) ultrasound is transmitted through various materials for the detection of discontinuities such as flaws or cracks [3]. In medical applications, ultrasonic reflections are the basis of forming images underneath the skin. Although there is an extensive body of data related to these types of applications, the high-frequency (and high-resolution) transducers designed to operate in these regimes are costly and therefore may be impractical for the 


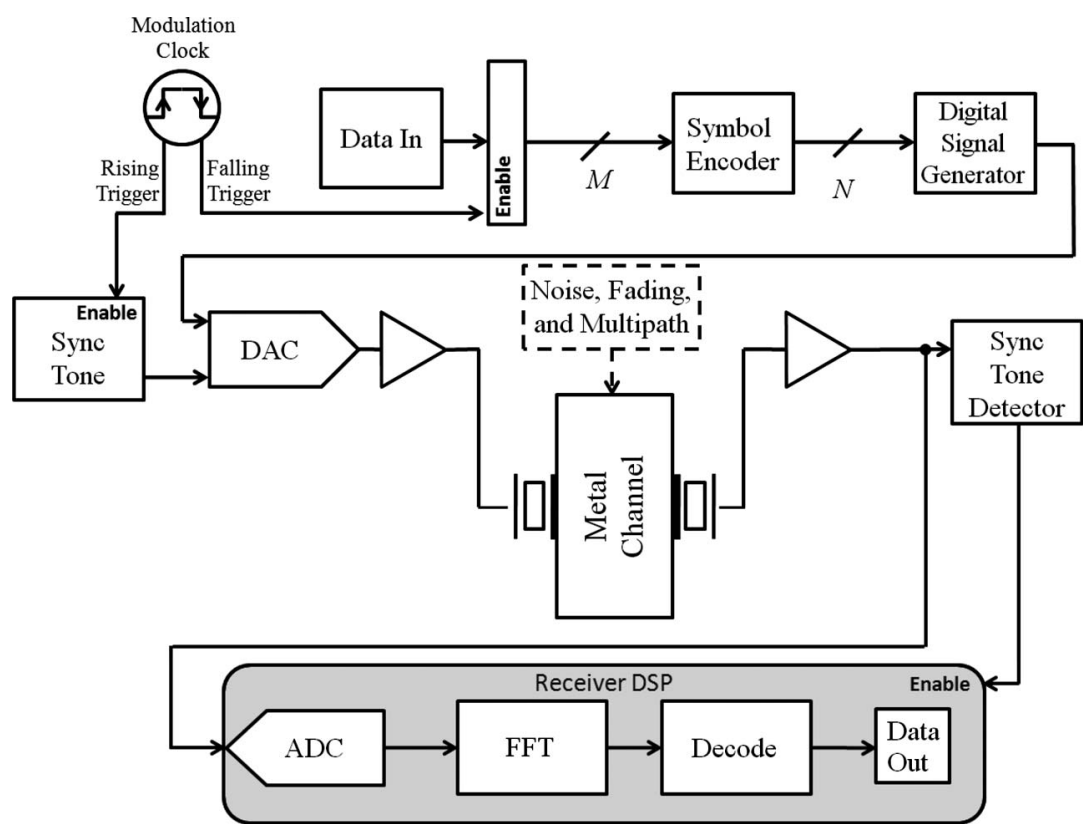

Fig. 1. System block diagram.

types of application domains where ultrasonic communications would be employed. Furthermore, high-frequency ultrasound has limited transmission range through metal.

Recently, studies involving ultrasonic communication through water, air, and metallic mediums have begun to appear. Underwater telemetry has been examined by several authors, including Baggeroer [5] and Stojanovic [6], and underwater communication researchers have found that FSK and multitone FSK (MFSK) provide robust modulation schemes, as in [7]-[9]. To briefly elaborate, Scussel et al. [8] used 1-of-4 MFSK and Hadamard MFSK to transmit up to $2400 \mathrm{~b} / \mathrm{s}$. Another research on underwater communication with autonomous underwater vehicles was conducted at Florida Atlantic University [9]. This research used multifrequency shift keying to communicate in shallow water up to $5000 \mathrm{~m}$.

Air mediums have been studied by several authors, and a few examples are given here. Short-range (1-2 m) ultrasonic communication through air has been studied in the context of quadrature phase-shift keying (QPSK) for improved data rates [10]. The researchers used capacitive ultrasonic transducers to transmit data through air using QPSK. They were able to achieve reliable data transmission of up to $1 \mathrm{~m}$ under laboratory conditions. Research involving through-air ultrasonic communication was performed by S. Holm [11]. Transmission up to $20 \mathrm{~m}$ was obtained using FSK. Research was also conducted at Oak Ridge National Laboratories in 1993 and further in 1999, where a successful demonstration system was built to communicate using ultrasound in air [12]. Oak Ridge National Laboratories also experimented with various types of pipes, including galvanized, copper, and PVC [Ibid]. For this demonstration system, a laptop with a LabView Virtual Instrument at each end of the communication channel was employed.

A relatively small number of experiments have been conducted using ultrasonic communication through metallic structures. Sakuma et al. experimented with communicating through metal gas pipe lines using ultrasonics [13]. These signals were transmitted using a frequency-hopping scheme, and modulation schemes of differential phase-shift keying and quadrature amplitude modulation were compared. Saulnier et al. worked to communicate ultrasound through a 6-in steel wall [14]. The research in [14] describes three approaches for communicating through the steel block: a double-hop approach, a reflectedpulse approach, and an approach consisting of a hybrid between the two. In another metallic application, Tomlinson et al. in 2007 designed an ultrasonic communication system to operate within an aircraft's airframe and across the outside of a steel shipping container [15]. However, this prior research related to shipping containers was limited in several ways.

1) It did not consider communication from within a container to the outside of a container.

2) It did not consider communication among stacked containers.

3) It did not consider two-way communication.

4) It did not proceed to the system-prototyping stage.

The subsequent sections of this paper describe the design and implementation of an ultrasonic communication system for stacked shipping containers and are organized as follows: Section II discusses the required methods to effectively communicate through the steel channel. Section III describes the implementation of the system design and optimizations for the design. Lastly, Section IV discusses future work and our conclusions. This paper builds on the ultrasonic work that we presented in [16]. In particular, we offer additional laboratory data and expanded discussions on the sampling strategy, optimization approaches, new hardware approaches, and accuracy studies.

\section{METHODS AND APPROACH}

To solve the communication problem, the team's design is represented by the block diagram in Fig. 1. The modulation clock triggers the transmission of the synchronization tone and 


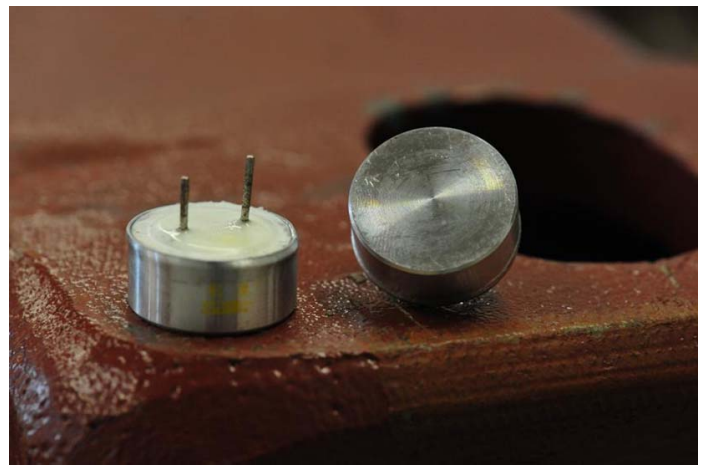

Fig. 2. Employed transducers on the end of a corner post. The transducer face is slightly larger than the diameter of a quarter.

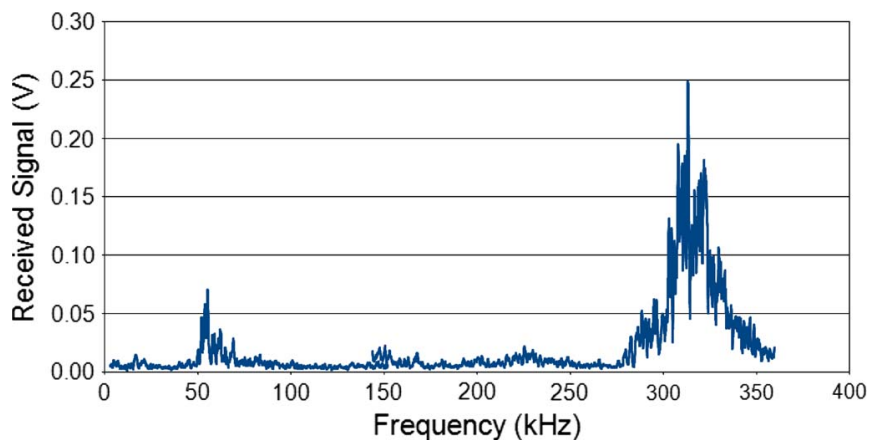

Fig. 3. Received signal voltage versus frequency for a pair of transducers using a swept sinusoid of constant amplitude.

data. After a sync tone is transmitted to initiate communication between the transmitter and the receiver, the transmitter encodes and modulates the data before sending them to the digitalto-analog converter (DAC). The receiver then samples the modulated signal with the analog-to-digital converter (ADC), performs fast Fourier transform (FFT), and decodes the signal in order to output the data.

To engineer a robust system, it was necessary to characterize the piezoelectric transducers and the steel channel. An MFSK modulation scheme was chosen to combat severe fading that arises from transmitting ultrasound in a steel communication channel. In the following three subsections, we discuss characterization of the ultrasonic transducers, empirical analysis of the steel channel, and MFSK modulation.

\section{A. Characterization of Piezoelectric Ultrasonic Transducers}

A photograph of the transducers used in this study is shown in Fig. 2. These were purchased from Kobitone Audio [17] and were designed to have a center frequency of $40 \mathrm{kHz}$, a bandwidth of $1 \mathrm{kHz}$, and a sound pressure level of $115 \mathrm{~dB}$ at $40 \mathrm{kHz}$ with a reference of $0.0002 \mu \mathrm{bar}$. Characterization of the transmission efficiency of the transducer pair used in this experiment was determined over a wide frequency spectrum. Data gathered by pulsing one transducer-with a receiving transducer clamped face to face-were used to produce the empirical frequency response curve in Fig. 3. From the figure, it is clear that the employed transducers exhibit two major regions of resonance. The first occurs around the manufacturer-

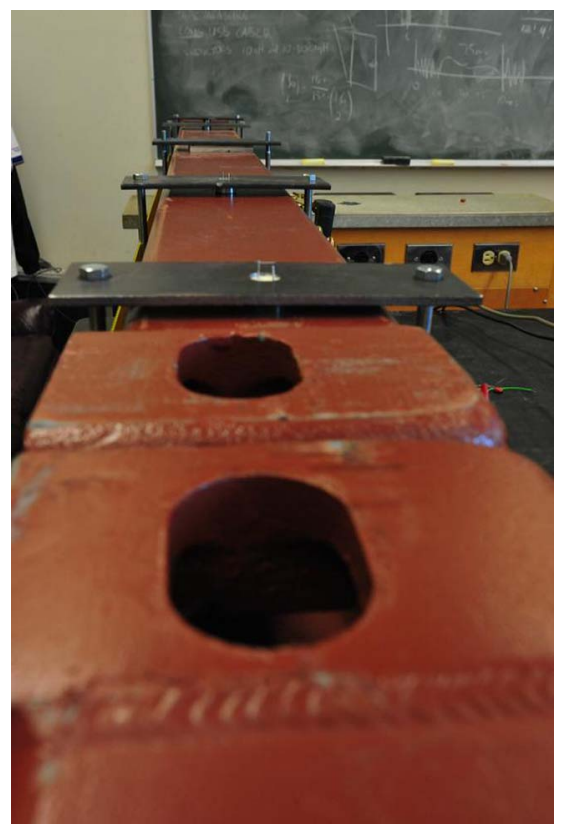

Fig. 4. Corner post setup showing two abutted corner posts held in compression by a hand hoist and yellow nylon strapping (faintly visible). Four transducers mounted along the length of the corner post are shown.

specified resonant frequency of the device, at about $40 \mathrm{kHz}$ [17]. The second occurs on a band beginning near $260 \mathrm{kHz}$ and extending to approximately $330 \mathrm{kHz}$. This second band is well displaced from the nominal $40-\mathrm{kHz}$ center frequency of the transducers and produces a better voltage response by a wide margin.

\section{B. Empirical Channel Evaluation}

Two shipping container corner posts were acquired for demonstrating the communication system developed. The corner post setup is shown in Fig. 4, which shows two corner posts horizontally lying on a support structure and held in compression with a hand hoist and nylon strapping. Importantly, for the shipping container application, it was discovered that ultrasonic signals can be transmitted from one corner post and received on a second corner post abutted to the first. In actual use, corner posts would be associated with containers. Two stacked containers would be supported by four pairs of abutted corner posts.

With the steel channel established, empirical analysis was performed to find an optimal way to transmit and receive digital data. Three aspects of channel evaluation are described through the remainder of this subsection.

1) Wave Fundamentals and Channel Characterization: Acoustic waves are used for a number of applications, as noted in the introductory section. Their use for communications has primarily been in underwater applications. The behavior of the waves in these applications has some distinct differences from waves traveling through solid mediums. Waves traveling through water are generally caused by compressions and rarefactions of the water. Compression refers to a region of high mass density, whereas rarefaction refers to a region of low particle density. As the wave propagates, these regions 


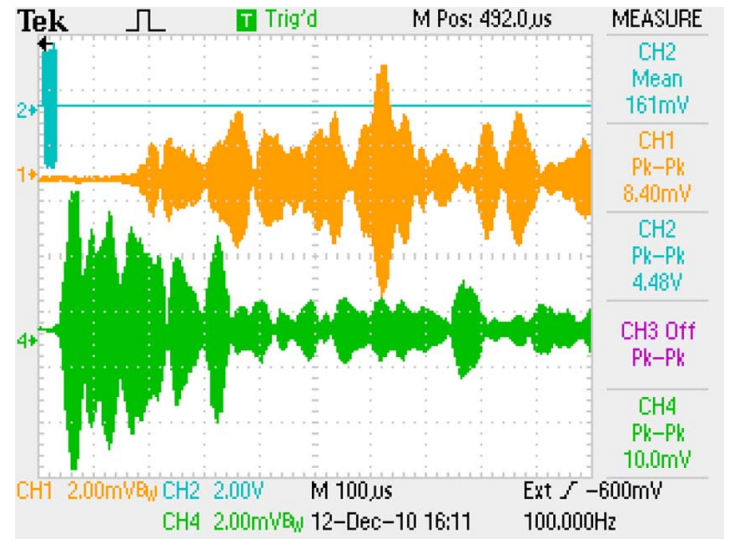

Fig. 5. Screen shot of a $300-\mathrm{kHz}$ pulse in blue being transmitted and received by two other transducers in orange and green. The orange transducer receives a signal that is very different from the green's received signal due to the many wave modes interacting in the steel channel. However, all received signals have no Doppler shift.

appear in sequence with one another-like a set of dominoes. The boundaries consist of the surface where the air meets the water and the floor of the water container. Underwater waves spread in both time delays and Doppler shifts. In contrast, acoustic waves traveling through a solid medium, such as steel, have many modes of travel. These include longitudinal, transverse, surface, and plate waves. Longitudinal waves travel parallel to the direction of the wave, whereas shear or transverse waves travel perpendicular to the direction of the wave. Surface waves have an elliptical orbit. Plate waves consist of Love and Lamb waves and travel in thin material that is only a few wavelengths thick. Love waves move shear to their bounding surface and change velocity with respect to its frequency. Lamb waves have infinite modes of propagation and are dependent on the entry angle, frequency, and structural geometry of the channel [18].

The purpose of this research was not to create a complex model of a metallic communication channel but to find a practical way to communicate through a metallic channel. We have empirically studied the channel and from these studies can predict channel behavior with sufficient fidelity. In Fig. 5, we can see complex signals arise from a $26-\mu$ s pulse. These signals are created through the multipath of the different wave modes, as described in the previous paragraphs. While there is strong constructive and destructive interference due to the multipath, there is no Doppler shift in the signal, as each signal contains the carrier frequency of the pulse signal, which, for this study, was set at $300 \mathrm{kHz}$.

2) Reciprocity: Reciprocity or symmetry of transmission between two points on the channel is an important characteristic of the channel. It ensures that communication in both directions can use the same transmission tones. Evidence for reciprocity was experimentally investigated by placing transducers at the five locations illustrated in Fig. 6. One transducer was chosen as the transmitter, with another as the receiver. Eight tones were transmitted, and the received signal's frequency response was captured. Then, the transmitter and receiver leads were switched, without moving the transducers, and the experiment

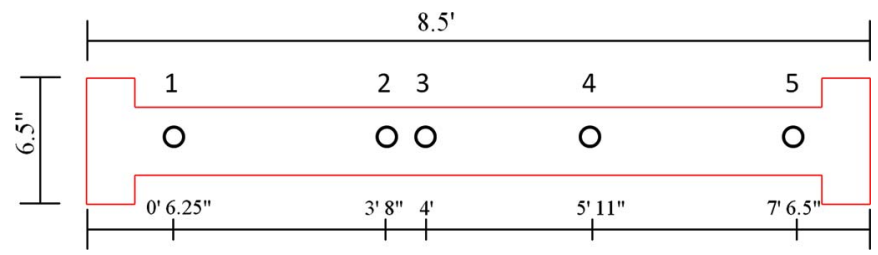

Fig. 6. Empirical setup of the channel. Each circle represents the placement of an ultrasonic transducer on the post.

was repeated. There was strong evidence that reciprocity exists in this real-world channel.

3) Spatial Relevance: Spatial distance between transducers was varied to determine its impact on signal transmission. This experiment was performed by choosing a point along the channel to transmit the data and capturing the received signal from several different points to simulate locations of possible module placement. Although transmission was generally consistent, it was noted that there were changes in the frequency response dependent on the distance between the transmitting and the receiving transducers. This is due to the multipath that arises from the steel channel. The multipath of the signal may create destructive or constructive interference among specific frequencies, depending on the spacing of the transmitting transducer, receiving transducer, and the corner posts. Therefore, some frequencies may transmit well in some locations, whereas, in other locations, they may be attenuated. Fig. 7 shows the responses of two receiving transducers at different distances from the same transmitter. From the image on the left, the transmitted tones $f_{0}$ and $f_{2}$ were not received well, whereas the image on the right shows that $f_{0}$ and $f_{2}$ were received better but $f_{3}$ has been attenuated. In summation, MFSK tones transmit well in the steel channel, but a training sequence will need to be performed in a fielded system to map out optimal transmission tones. All other experiments performed here are done under the assumption that the frequency response has been mapped for the specific transducer locations used.

\section{Modulation Approach: MFSK}

MFSK, which is a generalization of FSK, is known to be well suited for fading channels [19]. Ultrasonic signals transmitted through mediums such as steel or water are subjected to extreme fading effects. In the case of transmitting ultrasound through a steel beam, fading is primarily due to two phenomena: 1) multipath effects due to multiple reflections from channel boundaries and 2) the effect of multiple waves traveling through the channel at different velocities [15].

Traditional FSK represents different symbols with individual sinusoidal tones. Thus, for a pool of $N$ tones, FSK can encode at most $N$ symbols. MFSK uses different combinations of multiple frequencies (summed tones) to encode symbols [19]. The MFSK scheme in which exactly $Q$ tones are used at once is known as $Q$-tone MFSK. Let $N$ denote the set of tones available for defining symbols, with $Q$ being the number of tones in each $Q$-tone MFSK symbol. It follows that $Q$-tone MFSK can encode $\left(\begin{array}{l}N \\ Q\end{array}\right)$ symbols. $\left(\begin{array}{l}N \\ Q\end{array}\right)$ defined by $N ! /(Q !(N-$ $Q) !)$ is interpreted as the number of different combinations 

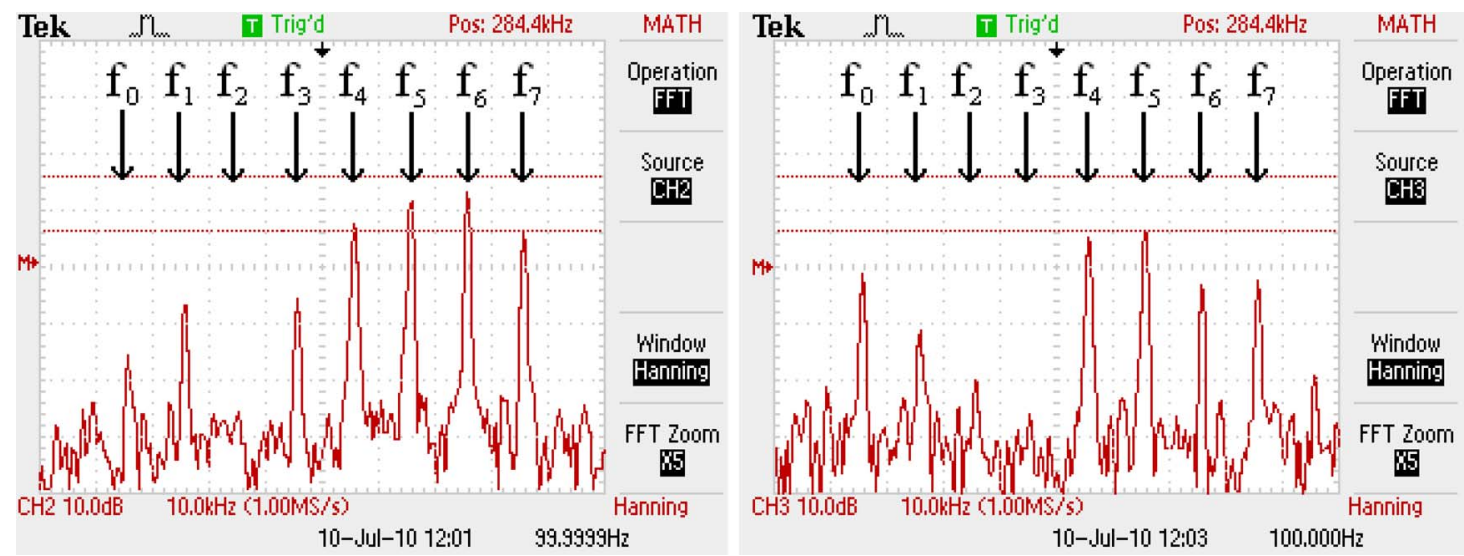

Fig. 7. Two transducers receiving the same transmitted signal at different locations.

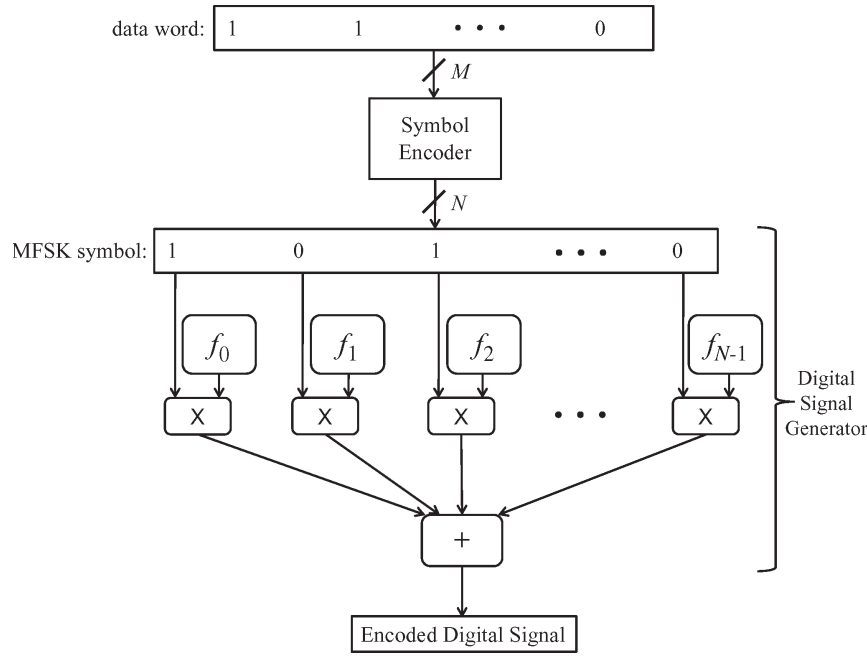

Fig. 8. MFSK encoding scheme.

of $Q$ elements from an $N$-element set. Using $Q$-tone MFSK is advantageous in that the system can utilize the full bandwidth of the channel while maintaining low power with relatively high bits per transmission. On the transmission side, an $M$-bit data word is input to a symbol encoder module (where $Q<M<N)$, which produces an $N$-bit symbol, as shown in Fig. 8. For $Q$-tone MFSK, exactly $Q$ of these $N$ bits are unity, which correspond to selected tones of the symbol.

In principle, an MFSK receiver employs a bank of matched filters with center frequencies tuned to each of the $N$ tones. For the implementation described, the received signal is digitized, and FFT is performed on the receiver's digital-signalprocessing (DSP) chip. The receiver thresholds the magnitude of the values from the FFT frequency bins associated with the $N$ tones. If exactly $Q$ tones exceed prescribed thresholds, then the corresponding symbol is detected. Otherwise, the receiver declares a transmission error. Experiments were conducted with $N=23$ and $Q=8$. The frequency band was from 260 to $320 \mathrm{kHz}$. Each of the frequencies $f_{k}$, where $k=0$ to $N-1$, was selected by using only the frequencies that returned the highest amplitude response while maintaining a distance of at least $1.5 \mathrm{kHz}$ apart. In this experimental system, $\left(\begin{array}{c}N \\ Q\end{array}\right)$ is $\left(\begin{array}{c}23 \\ 8\end{array}\right)$, which gives a symbol set of 490314 symbols. Thus, data words of size $M=18$ can be encoded because $2^{18}=262144<490314$.

\section{System Design}

The team's initial focus was on designing and constructing a receiver to capture and decode MFSK symbols transmitted down a steel beam. On the transmission side, an arbitrary signal generator, i.e., Agilent $81150 \mathrm{~A}$, and an amplifier circuit produced and transmitted the MFSK symbols. The current receiver includes an amplifying and biasing circuit, an LM567 tone decoder, and a DSP chip. The amplifying and biasing circuit was used to amplify the received signal in order to utilize the full dynamic range of the DSP chip's onboard ADC. The circuit had a signalto-noise ratio of $27 \mathrm{~dB}$ and a gain of $600 \mathrm{~V} / \mathrm{V}$. This amplifies the signal from $5 \mathrm{mVpp}$ to $3 \mathrm{Vpp}$ on a 1.5 -V bias. The LM567 chip was chosen because it provides an easy and inexpensive way to detect the synchronization tone. Instead of the DSP chip constantly sampling and filtering to detect synchronization, the DSP chip is instead put into a low-power idle state until the sync tone is detected by the LM567. This is key to keeping the communication module as energy efficient as possible.

Initially, a DSP chip was chosen for its potential to rapidly compute the spectrum of the received signal via FFT. The Texas Instruments TMS320LF2407A DSP chip was chosen for this project, based on its low-cost aspects, features, and low power consumption. The F2407A is a 16-bit fixed-point DSP chip that is capable of operating at speeds up to 40 million instructions per second. The F2407A has an internal flash memory (32 KB), which allows for rapid prototyping and eliminates costly offchip memory. This particular DSP chip also has an onboard ADC with a 500-ns conversion time. This feature mitigated the need for an additional off-board converter. The lower shaded portion of Fig. 1 depicts the functionality performed by the DSP chip. The sync tone is sent at the beginning of a transmission cycle, which corresponds to the modulation clock rising to its high state. When the modulation clock falls to its low value at half the clock period, the MFSK symbol is transmitted. Fig. 9 shows the actual scope traces of the transmission of the sync tone, followed by the MFSK signal. As the MFSK signal travels down the channel, the signal degrades and fades but is eventually picked up by the receiving transducer. This signal is then amplified to $3 \mathrm{Vpp}$ and sent to a biasing circuit, as well as the tone decoder.

Fig. 10 provides a detailed timing diagram showing how the sync tone (in red) is used to synchronize the receiver with the 


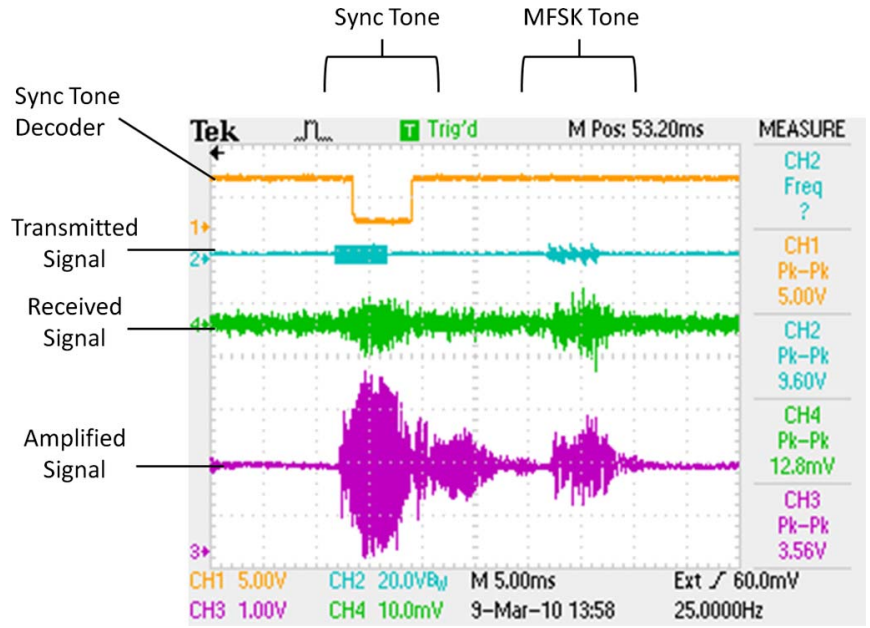

Fig. 9. Signals from top to bottom: LM567 output trigger, transmitted MFSK signal, received signal, and amplified signal. The first signal (on the left) is the sync tone; the second smaller signal is the MFSK waveform.
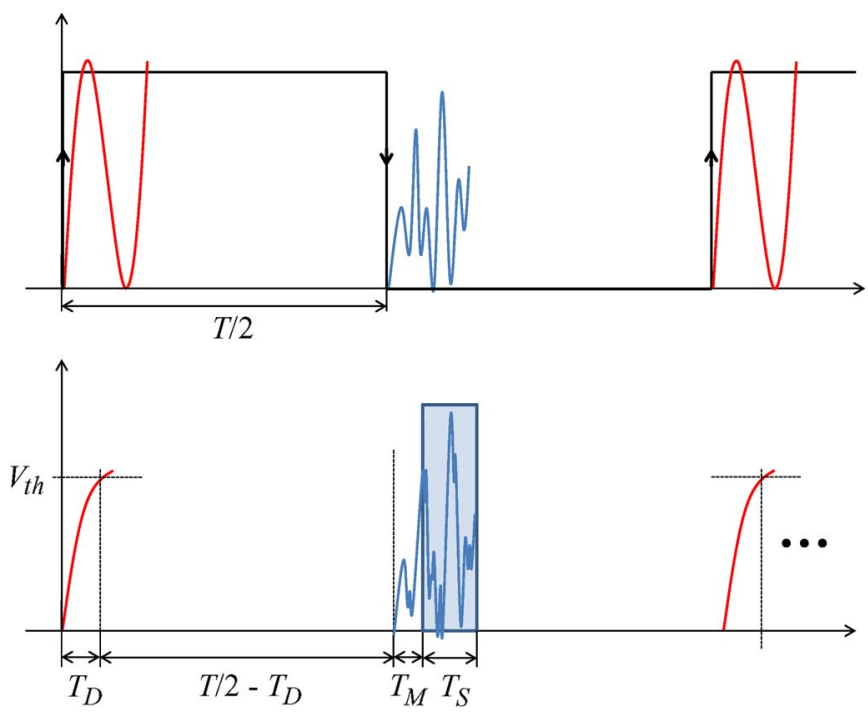

Fig. 10. Timing diagrams. (Top) Transmission of the sync tone (red) and the signal (blue). (Bottom) Detection and sampling of the data.

transmitted signal (in blue). When the sync tone is transmitted, the LM567 detects the tone after a (predictable) delay time of $T_{D}$. The value of $T_{D}$ includes the time for the signal to propagate down the channel and trigger the LM567 chip. Once triggered, the LM567 pulls down a general-purpose I/O (GPIO) on the F2407A. The GPIO wakes up the F2407A from its power-efficient mode and activates the necessary peripherals to start processing the received ultrasonic signal. The F2407A waits for a specified time of $T / 2-T_{D}+T_{M}$, where $T_{M}$ represents a safety margin to ensure that the DSP chip takes samples near the center of the received signal pulse. The DSP samples at a rate of $165 \mathrm{kHz}$ for a total sampling time of $T_{S}$ and collects 512 samples. The time interval in which sampling occurs is depicted by the blue shaded region in Fig. 10. Once 512 samples are collected, a radix-2 FFT is performed on the samples. The magnitude of the FFT is then compared with a threshold at specific frequency bins corresponding to the frequency tones of the MFSK modulation.

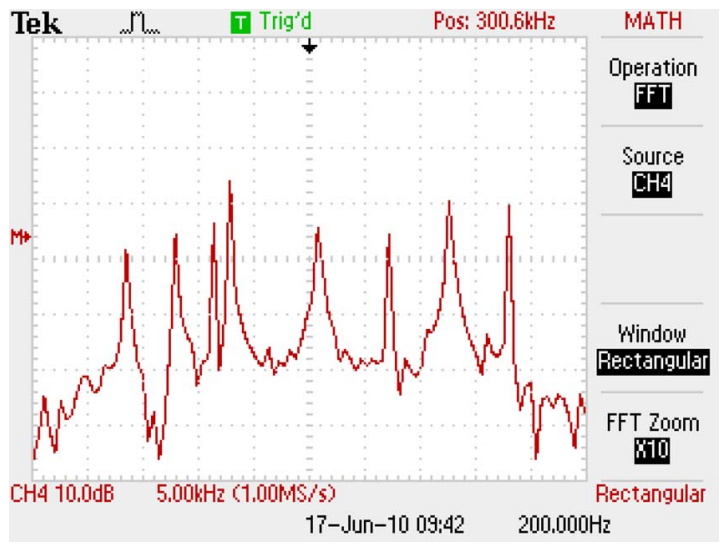

Fig. 11. Experimentally received noncoherent signal consisting of eight tones.

\section{A. Implementation}

Two experimental scenarios were conducted: the first was with a transmitter and a receiver on the same corner post, and the second was with the transmitter and the receiver on separate abutted corner posts. MFSK symbols were successfully transmitted with few errors for each case. Due to the relatively slow performance of the inexpensive DSP chip employed for this initial study, the symbol rate achieved was only $20 \mathrm{~Hz}$. With 18 bits of data encoded in each transmission of $\left(\begin{array}{c}23 \\ 8\end{array}\right) Q$-tone MFSK, the effective bit rate achieved was $360 \mathrm{~b} / \mathrm{s}$. However, future implementations will utilize a faster DSP chip enabling transmission rates of at least $100 \mathrm{~Hz}$, resulting in bit rates of about $1800 \mathrm{~b} / \mathrm{s}$.

\section{B. Sampling Approach}

By definition, bandpass sampling is a special form of undersampling that translates a high-frequency bandpass signal to a baseband frequency [20]-[24]. This concept provides a technique of reduced sampling speeds, which directly translates to reduced power consumption (as the authors have been able to demonstrate in [25]). For this ultrasonic project, this approach enabled the sampling of the received signal to be only $165 \mathrm{kHz}$, which is just slightly over one-half the maximum tone frequency of $320 \mathrm{kHz}$. Had this undersampling procedure not been utilized, then a high-speed ADC sampling rate would have been required.

In addition, the transmitted tones were chosen with the following: 1) Each tone was designed to fall within a specific frequency bin of the FFT, and 2) an integer number of periodic cycles of each tone within of the sampled waveform could be collected and processed by a data frame of the FFT within the DSP chip. These two constraints, which are also known as coherency, form a method that increases the spectral resolution of an FFT and eliminates the need for window sampling when conditions 1 and 2 are met. Experiments on coherency, within the bandpass sampling framework, were performed with $M$-ary orthogonal signals, where $M$ was chosen to be eight. Figs. 11 and 12 show that coherency significantly increases the resolution at each frequency and therefore makes them easier to detect. 


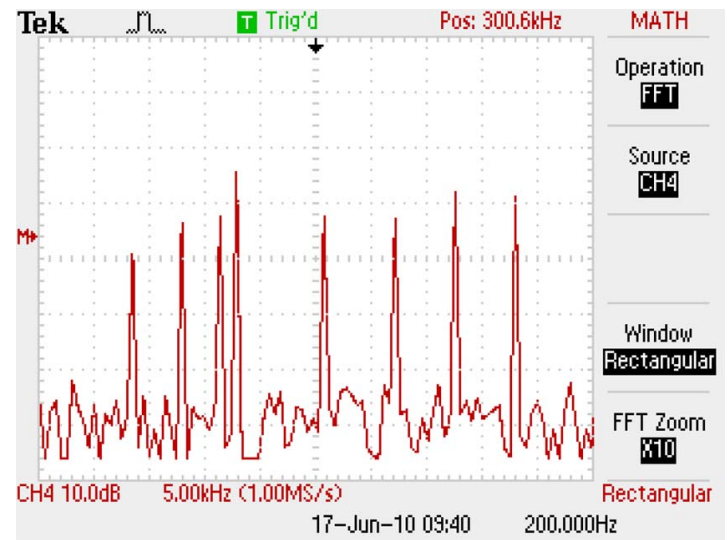

Fig. 12. Experimentally received coherent signal consisting of eight tones.

\section{Handshaking}

While different transmitter/receiver locations along the channel generally have similar frequency responses, there is a potential for some frequencies to be emphasized and others to be attenuated. Handshaking will enable each pair of transmitter/ receiver modules to accurately represent and utilize the frequency response of the channel, which depends on the relative location of the transducers. We can map out the most efficient tones to be used for communication by making use of the reciprocity property of our channel, as described in Section II-B2. This process will begin with the transmitter sending out a sync tone to activate the receiver. The receiver will then transmit a set of $Q$ tones separated by the band of the useful spectrum $f_{\mathrm{Hi}}-f_{\mathrm{Lo}}$, which is divided by the number of tones $Q$, i.e., $\left(f_{\mathrm{Hi}}-f_{\mathrm{Lo}}\right) / Q$. The transmitter will keep track of the received $Q$ tones and their magnitude in the FFT. The receiver will next increment each of the $Q$ tones up the spectrum by $f_{\text {inc }}$, where $f_{\text {inc }}$ is defined by the size of the smallest frequency bin location of the FFT. Once the entire spectrum has been covered, the transmitter will then send out a series of transmissions using the $N$ best received tones that are at least $f_{\min }$ apart, where $f_{\min }$ denotes the minimum frequency distance that can be accurately seen by the FFT and can be coherently sampled, as stated in Section III-B. Once the $N$ tones have been sent, the transmitter will send out a sync tone to designate the end of transmission. Through this handshake, the receiver and transmitter will be able to represent $\left(\begin{array}{l}N \\ Q\end{array}\right)$ symbols. The time that this handshake will take with our current apparatus is a little over $1 \mathrm{~s}$. This assumes that our frequency band is from 260 to $330 \mathrm{kHz}$, the transmission rate is $20 \mathrm{~Hz}, f_{\mathrm{inc}}=322 \mathrm{~Hz}$, and $Q=8$.

\section{Equalization Studies}

Equalization enables the utilization of frequencies that would not otherwise be easily detectable and therefore increases the data rate at which we can send our signal. As seen in Fig. 3, the band used for transmission is not completely flat. We therefore used equalization as a means to flatten the magnitude at which the frequencies are received. This is done by weighing each frequency in the MFSK symbol and then normalizing the symbol before it gets transmitted. This will place more emphasis on specific frequencies while maintaining full utilization of

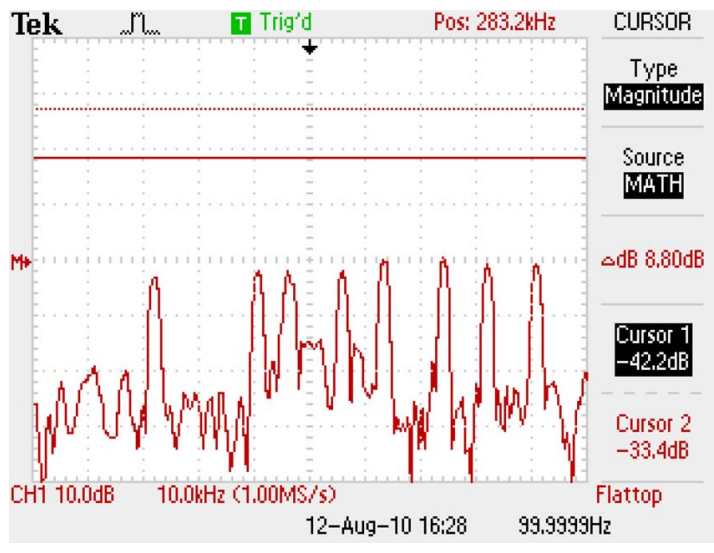

Fig. 13. Example of received symbol containing eight equalized tones.

the DAC. Fig. 13 shows that we can get close to having the frequency peaks of all received signals at the same magnitude, which greatly increases the number of coherently transmitted tones. As the word was increasingly weighted, more energy was needed to transmit the MFSK symbol to an optimal voltage for the receiving ADC. This is because, as more energy is put into a set of less efficient frequencies, the other tones will receive less energy; therefore, the symbol arrives at a lower voltage. Equalization is performed after two modules complete the handshaking process.

\section{Conclusion and Future Plans}

This paper has described the design of an MFSK communication system based on ultrasonic signals that are transmitted through steel shipping container corner posts and utilize lowcost transducers. We have demonstrated the ability to reliably encode and transmit 18 bits of data per modulated MFSK symbol. Experiments conducted have shown that inexpensive transducers are capable of both transmitting and receiving ultrasonic signals through steel with a relatively high signal-to-noise ratio and low transmit power. Empirical studies have shown that handshaking can be performed for accurate base tone selection. Equalization can also be performed to enhance reception characteristics. The low-power aspects of the transmitter/receiver have been enhanced by leveraging undersampling techniques to implement a bandpass sampling strategy, enabling the use of low-power and low-sampling-rate ADCs. Our current system uses inexpensive parts to build a working receiver, achieving a data rate of about $360 \mathrm{~b} / \mathrm{s}$. Currently, our data transmission is limited by how quickly the low-cost F2407A can perform FFT. With an upgraded DSP chip, the potential to more quickly receive data will be limited only by the intersymbol interference of the channel. Future research will evaluate and develop specific MFSK symbol encodings, as well as communication protocols. A complete transmitter/receiver module that is capable of sensing and storing data from an array of sensors will also be constructed. This future unit will have a more powerful and more efficient processor that is capable of performing FFT much faster than the current processor. Evaluation for the optimal set of $Q$ tones will be performed. For the shipping container application, experiments will be conducted on fully constructed containers. 


\section{REFERENCES}

[1] A. Sabatini, "A digital-signal-processing technique for ultrasonic signal modeling and classification," IEEE Trans. Instrum. Meas., vol. 50, no. 1, pp. 15-21, Feb. 2001.

[2] D. Ensminger and F. Stulen, Ultrasonics: Data, Equations, and Their Practical Uses, 1st ed. Boca Raton, FL: CRC Press, 2008

[3] D. Ensminger, Ultrasonics, 2nd ed. New York: Marcel Dekker, 1988.

[4] V. Kaftandjian, O. D. Y. Zhu, and D. Babot, "The combined use of the evidence theory and fuzzy logic for improving multimodal nondestructive testing systems," IEEE Trans. Instrum. Meas., vol. 54, no. 5, pp. 19681977, Oct. 2005

[5] A. Baggeroer, "Acoustic telemetry-An overview," IEEE J. Ocean. Eng., vol. OE-9, no. 4, pp. 229-235, Oct. 1984.

[6] M. Stojanovic, "Recent advances in high-speed underwater acoustic communications," IEEE J. Ocean. Eng., vol. 21, no. 2, pp. 125-136, Apr. 1996.

[7] D. Wax, "MFSK-The basis for robust acoustical communications," in Proc. OCEANS, Sep. 1981, pp. 61-66.

[8] K. Scussel, J. Rice, and S. Merriam, "A new MFSK acoustic modem for operation in adverse underwater channels," in Proc. MTS/IEEE Conf. OCEANS, Oct. 1997, vol. 1, pp. 247-254.

[9] L. LeBlanc, M. Singer, P.-P. Beaujean, C. Boubli, and J. Alleyne, "Improved chirp FSK modem for high reliability communications in shallow water," in Proc. MTS/IEEE Conf. Exhib. OCEANS, 2000, vol. 1, pp. 601-603.

[10] C. Li, D. Hutchins, and R. Green, "Short-range ultrasonic communications in air using quadrature modulation," IEEE Trans. Ultrason., Ferroelectr, Freq. Control, vol. 56, no. 10, pp. 2060-2072, Oct. 2009.

[11] S. Holm, "Airborne ultrasound data communications: The core of an indoor positioning system," in Proc. IEEE Ultrason. Symp., Sep. 2005, vol. 3, pp. 1801-1804.

[12] H. Haynes and M. Akeman, "Final report, ultrasonic communication project, phase 1, fy 1999," Eng. Technol. Div., Oak Ridge Nat. Lab., Oak Ridge, TN, Jun. 2000.

[13] H. Sakuma, K. Nakamura, and S. Ueha, "Two-way communication over gas pipe-line using multicarrier modulated sound waves with cyclic frequency shifting," Acoust. Sci. Technol., vol. 27, no. 4, pp. 225-232, 2006.

[14] G. Saulnier, H. Scarton, A. Gavens, D. Shoudy, T. Murphy, M. Wetzel, S. Bard, S. Roa-Prada, and P. Das, "Through-wall communication of low-rate digital data using ultrasound," in Proc. IEEE Ultrason. Symp., Oct. 2-6, 2006, pp. 1385-1389.

[15] H. Tomlinson, J. Daton, E. Nieters, and F. Ross, "Ultrasound communication system and related methods," U.S. Patent Application US 2007/ 0167133 A1, Jul. 19, 2007.

[16] T. Hosman, M. Yeary, J. Antonio, and B. Hobbs, "Multi-tone FSK for ultrasonic communication," in Proc. IEEE Int. Instrum. Meas. Technol. Conf., May 3-6, 2010, pp. 1424-1429.

[17] Kobitone Audio Company, Tech. Rep. Ultrasonic Transducers Part Number: 255-400er25-rox, 255-400et25-rox, Data Sheet, Arlington, TX, 2007.

[18] Z. Su and L. Ye, Identification of Damage Using Lamb Waves: From Fundamentals to Applications, 1st ed. New York: Springer-Verlag, 2009.

[19] C. Luo, M. Medard, and L. Zheng, "On approaching wideband capacity using multitone FSK," IEEE J. Sel. Areas Commun., vol. 23, no. 9, pp. 1830-1838, Sep. 2005.

[20] D. Akos, M. Stockmaster, J. Tsui, and J. Caschera, "Direct bandpass sampling of multiple distinct RF signals," IEEE Trans. Commun., vol. 47, no. 7, pp. 983-988, Jul. 1999.

[21] C. Tseng and S. Chou, "Direct downconversion of multiband RF signals using bandpass sampling," IEEE Trans. Wireless Commun., vol. 5, no. 1, pp. 72-76, Jan. 2006.

[22] R. Vaughan, N. Scott, and D. White, "The theory of bandpass sampling," IEEE Trans. Signal Process., vol. 39, no. 9, pp. 1973-1984, Sep. 1991.

[23] N. Wong and T. Ng, "An efficient algorithm for downconverting multiple bandpass signals using bandpass sampling," in Proc. IEEE Int. Symp. Circuits Syst., Jun. 2001, vol. 3, pp. 910-914.

[24] D. Linden, "A discussion of sampling theorems," Proc. IRE, vol. 47, no. 7, pp. 1219-1226, Jul. 1959

[25] M. Yeary, J. Meier, R. Kelley, and R. Palmer, "Compact digital receiver development for radar based remote sensing," in Proc. IEEE Int. Instrum. Meas. Technol. Conf., May 2008, pp. 1761-1765.

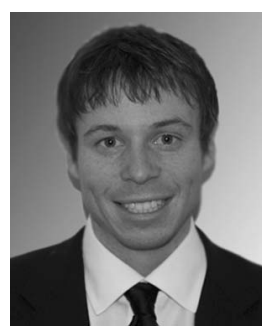

Thomas Hosman received the B.S. degree in electrical engineering, in 2009, from the University of Oklahoma, Norman, where he is currently working toward the M.S. degree in electrical engineering with the School of Electrical and Computer Engineering.

$\mathrm{He}$ plans to pursue a career in the industry as a hardware design engineer after graduation. His research interests include hardware design, working with sensors and communication transceivers.

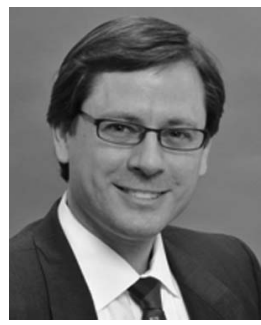

Mark Yeary (S'95-M'00-SM'03) received the B.S (honors), M.S., and Ph.D. degrees from Texas A\&M University, College Station, in 1992, 1994, and 1999 , respectively.

Following his graduation in 1999, he continued his digital signal processing (DSP)-based research at Texas A\&M University and collaboratively worked with a variety of companies, including IBM, Raytheon, Cisco, Texas Instruments, and LockheedMartin. He is currently with the School of Electrical and Computer Engineering, University of Oklahoma (OU), Norman, where he has been recently named the endowed HudsonTorchmark Presidential Professor. While with OU, he has served as a Principle Investigator (PI) or Co-PI on federal projects sponsored by the National Aeronautics and Space Administration, the National Science Foundation, the Office of Naval Research, the National Oceanic and Atmospheric Administration (NOAA), the Air Force Office of Scientific Research, and various corporations. He has spent nine summers, from 2002 to 2010, with Raytheon. His research interests include DSP, as applied to customized DSP systems, tactical radars, and weather radars, with emphasis on hardware prototype development.

Dr. Yeary is a member of the Tau Beta Pi and Eta Kappa Nu honor societies. He was elected to be a National Science Foundation/Frontiers in Education 1998 New Faculty Fellow. He is a Fellow of NOAA's Cooperative Institute for Mesoscale Meteorological Studies. He is a licensed Professional Engineer. $\mathrm{He}$ has served as technical committee member for the IEEE International Instrumentation and Measurement Conference (I2MTC) on several occasions and as a technical co-chair for the I2MTC 2010. He currently serves as an I\&M Associate Editor and has been an Instrumentation and Measurement (I\&M) Society member for 15 years. By invitation, he was recently selected to participate in the U.S. National Academy of Engineering's Foundations of Engineering Education Symposium in 2010. He was the recipient of the IEEE 2005 Outstanding Young Engineer Award from the I\&M Society.

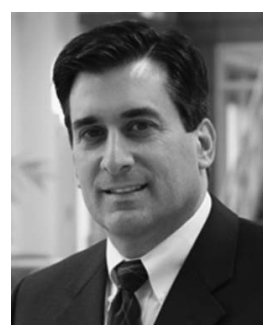

John K. Antonio (S'85-M'89-SM'98) received the B.S., M.S., and Ph.D. degrees in electrical engineering from Texas A\&M University, College Station.

From 1999 to 2006, he served as the Director and a Professor of computer science with the University of Oklahoma (OU), Norman. He is currently a Professor of computer science with the School of Computer Science, OU. Before joining OU, he was a faculty of electrical and computer engineering with Purdue University, West Lafayette, IN, and he was also a faculty of computer science with Texas Tech University, Lubbock. Numerous agencies and companies have supported his research over the years. He has been a Principal Investigator (PI) or Co-PI on more than 25 sponsored research projects totaling more than $\$ 2.5$ million. He has coauthored more than 100 publications and reports. He is the holder of a U.S. patent granted in 2010. His research interests include embedded high-performance computing, low-power and power-aware computing, reconfigurable computing, parallel and distributed computing, and cloud computing.

Dr. Antonio is a member of the Association of Computing Machinery and an elected member of the European Academy of Sciences. He has been Associate Editor for the IEEE TRANSACTIONS ON COMPUTERS. 